\title{
Effect of Dentin Bonding Agent on Intrapulpal Temperature during Fabrication of Provisional Restorations by a Direct Method: An In Vitro Study
}

\author{
Priyanka Gubrellay ${ }^{1}$, Milap Karia ${ }^{2}$, Kunal Talesara ${ }^{3}$, Chetan Sharma ${ }^{4}$, Shubhra Raghav ${ }^{5}$, Paranna Sujatha ${ }^{6}$
}

\begin{abstract}
Aim: To determine the effect of three different provisional restorative materials (PRMs) and application of dentin bonding agent (DBA) on intrapulpal temperature rise during fabrication of provisional crowns using a direct method.

Materials and methods: Three PRMs — polymethyl methacrylate resin (PMMA), bis-acrylic resin, and dimethyl methacrylate resin (DMMA)—were used in the study. Dentin bonding agent (Single Bond 2, 3M ESPE, Germany) was applied in a single layer or double layer as an insulating material. A total of 45 molars were prepared for complete coverage restoration with chamfer finish line and $1.5 \mathrm{~mm}$ axial and $2 \mathrm{~mm}$ occlusal reduction. After application of a single or double layer of DBA, provisional materials were polymerized according to the manufacturer's instructions. The temperature increase inside the pulp chamber was measured with a T-type thermocouple wire. The readings were averaged for each group to determine the mean value of temperature rise.

Results: Statistical analysis was performed with analysis of variance (ANOVA) followed by post hoc Tukey test $(a=0.05)$. The temperature rise varied according to the provisional restoration material used (PMMA $>$ DMMA $>$ bis-acrylic resin) $(p<0.001)$ and the single or double layer of DBA $(p<0.001)$. Polymethyl methacrylate resin produced a higher intrapulpal temperature.

Conclusion: Application of a double layer of DBA resulted in a significantly lower intrapulpal temperature.

Keywords: Dentin bonding agent, Dentinal tubules, Desensitizing agents, Provisional restoration.

The Journal of Contemporary Dental Practice (2019): 10.5005/jp-journals-10024-2624
\end{abstract}

\section{INTRODUCTION}

Provisional restorations can be defined as, "A fixed or removable dental prosthesis, or maxillofacial prosthesis, designed to enhance esthetics, stabilization and/or function for a limited period of time, after which it is to be replaced by a definitive dental or maxillofacial prosthesis". Their purposes are stabilization of occlusion, protection of the teeth and the periodontal tissues, function and esthetic enhancement, and provision of diagnostic information. ${ }^{2}$ Protection of the pulp is one of the principal biological requirements. ${ }^{3}$

There are two principal methods of fabricating such restorations, direct and indirect, with their own advantages and disadvantages. ${ }^{3}$ One of the major disadvantage is exposure of the tooth to the restorative material and the heat produced by polymerization of the resin. Since the dental pulp is particularly sensitive to elevated temperatures, the direct fabrication of provisional restorations can damage and traumatize the tooth. Damage from thermal changes includes various histopathologic changes of the pulp, such as ectopic odontoblasts and their destruction, cellular degeneration, burn reactions at the periphery of the pulp including formation of blisters, coagulation of protoplasm, expansion of liquid in the dentinal tubules, and vascular injuries with generalized or localized tissue necrosis, resulting in acute inflammation of pulp, irreversible pulpitis, pulp necrosis, and periodontal damage in severe cases. ${ }^{4}$

Thus, certain precautionary measures should be taken during fabrication of provisional restorations by a direct technique to minimize trauma to the pulpal tissue from the exothermic reaction of the resins. These include use of air and water coolant ${ }^{5,6}$ repeated removal and replacement of the template, using a matrix material that can dissipate heat rapidly ${ }^{7}$ or the application of desensitizing agents that occludes the dentinal tubules. ${ }^{8}$
1,2,4 Department of Prosthodontics, R.R. Dental College and Hospital,
Udaipur, Rajasthan, India
${ }^{3}$ Department of Conservative Dentistry and Endodontics, R.R. Dental
College and Hospital, Udaipur, Rajasthan, India
${ }^{5}$ Department of Public Health Dentistry, R.R. Dental College and
Hospital, Udaipur, Rajasthan, India
${ }^{6}$ Department of Pedodontics, Bharati Vidyapeeth Dental College and Hospital, Sangli, Maharashtra, India

Corresponding Author: Priyanka Gubrellay, Department of Prosthodontics, R.R. Dental College and Hospital, Udaipur, Rajasthan, India, Phone: +91 8003708816, e-mail: priyankagubrellay@gmail.com How to cite this article: Gubrellay P, Karia M, et al., Effect of Dentin Bonding Agent on Intrapulpal Temperature during Fabrication of Provisional Restorations by a Direct Method: An In Vitro Study. J Contemp Dent Pract 2019;20(8):947-951.

Source of support: Nil

Conflict of interest: None

During or after crown preparation, as many as 1-2 million dentinal tubules may be exposed, increasing the potential for postoperative sensitivity. ${ }^{8,9}$ Desensitizing agents occlude tubules by salt precipitation or resin deposition. Thus, if hypersensitivity is due to open dentinal tubules at the dentin surface, desensitizing agents may help in reducing the number of opened tubules, which in turn would reduce dentin hypersensitivity. Dentin adhesive systems, which seal the tubules from external stimuli, e.g., hot and cold, may prevent hydrodynamic fluid shifts, thereby stopping the stimulation of the nerve fibers and the resulting pain. ${ }^{8}$ Gurbulak et al. 
reported that the thickness of desensitizing agents affected the thermal changes and the double layer was more effective than the single layer. ${ }^{8}$

The purpose of this in vitro study was to test the effect of DBA when applied as a single or double layer on thermal changes in the pulp chamber using a thermocouple during direct fabrication of provisional restorations with three different PRMs.

\section{Materials and Methods}

The present study is an in vitro study conducted at the Department of Prosthodontics in M. S. Ramaiah Dental College, Bengaluru, Karnataka, India. Three PRMs - polymethyl methacrylate resin (DPI, Mumbai, India), bis-acrylic resin (Protemp ${ }^{\mathrm{TM}} 4$, 3M ESPE, Germany), and dimethyl methacrylate resin (Structure 2 SC, Voco, Germany) (Fig. 1)-were investigated in this study. Dentin bonding agent (Single Bond 2, 3M ESPE, Germany) was applied in a single or double layer as an insulating medium.

Forty-five extracted mandibular molars of average size and form were selected and stored in 10\% formalin. Teeth extracted due to periodontal conditions were used in the study. Teeth with attrition and cervical abrasion, former restorations, endodontic manipulation, and caries were not used in the study.

The specimens (45) were randomly assigned to three main groups according to the provisional material used; 15 teeth were included in each group:

Group A-polymethyl methacrylate resin (PMMA).

Group B-bis-acrylic resin.

Group C-dimethyl methacrylate resin (DMMA).

Each group was further divided into 3 subgroups:

Subgroup I (control)—without any insulating medium.

Subgroup II-with a single layer of DBA.

Subgroup III-with a double layer of DBA.

So, the study groups could be summarized as follows:

Group $A$, with subgroups $A 1, A 2$, and $A 3$.

Group B, with subgroups B1, B2, and B3.

Group C, with subgroups C1, C2, and C3.

Forty-five tests for each group were conducted. A total number of 135 tests were conducted.

The roots of mandibular molars were sectioned $2-3 \mathrm{~mm}$ below the cementoenamel junction with a carborundum disc. The pulp chamber was then cleansed of organic debris by irrigating with

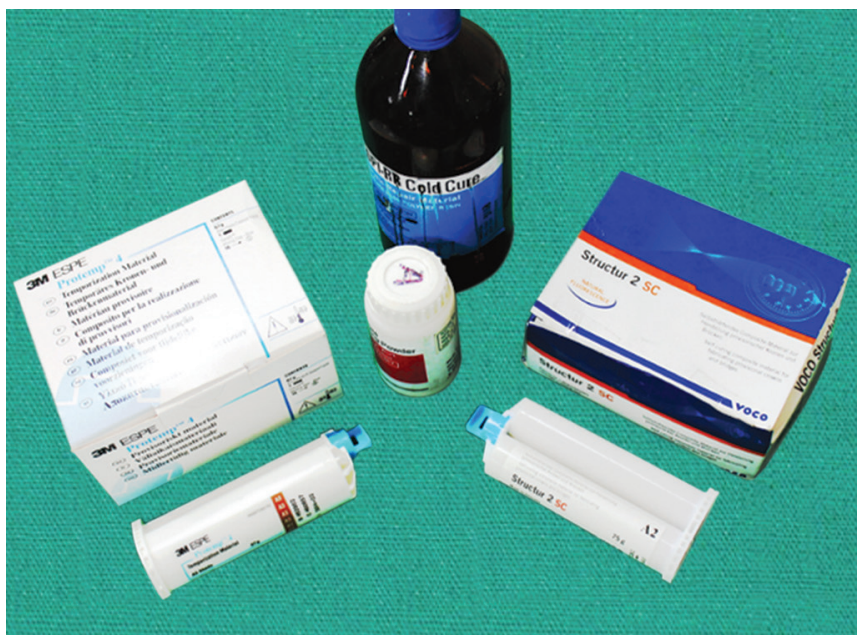

Fig. 1: Provisional restorative materials used in the study distilled water and 3\% sodium hypochlorite solution (VIP, Vensons India Pvt. Ltd, India), and the remnants of pulp tissue were cleaned off. The teeth were secured in a block of autopolymerising resin (D.P.I., Mumbai, India). A hole was made to provide entrance for the thermocouple wire into the pulpal chamber (Fig. 2). A T-type thermocouple probe (Heatcon Sensors Pvt. Ltd. Bengaluru, India) was positioned inside the pulp chamber of teeth and silver amalgam (DPI alloy, Fine Grain, Mumbai, India) was condensed around the thermocouple probe, filling the pulp chamber. Silver amalgam acts as a heat-conducting medium from dentin to the thermocouple probe.

Mandibular molars were prepared for complete coverage restoration with chamfer finish line and $1.5 \mathrm{~mm}$ axial and $2 \mathrm{~mm}$ occlusal reduction. The thermocouple lead was attached to a digital thermometer, which records intrapulpal temperature variation during temporization (Fig. 3). Each model was brought to a constant initial temperature by placing it in a preset water bath at $37^{\circ} \mathrm{C}$. Once the intrapulpal temperature was stabilized, the model was recovered and dried. It was immediately subjected to provisionalization.

Each of the 15 samples from group A was subjected to provisionalization with the putty index, without DBA application, which was the control test (A1). After insertion of the resin-filled matrix, the prepared tooth assembly was returned to the water bath to rule out the influence of environmental temperature on the test. The temperature was recorded using a thermocouple. Temperature monitoring was carried out till the resin was completely set according to the setting time recommended by the manufacturer, and the highest temperature attained was recorded.

After complete polymerization of the resin material, the index was removed. Then on the same tooth, a thin layer of DBA was applied all over the prepared surface with an applicator tip and was light-cured for 10 seconds. Then, the tooth was subjected to provisionalization. This was the second test (A2). For the third test, another layer of DBA was applied with an applicator tip on and was light-cured for 10 seconds. This was then subjected to provisionalization by a direct method (A3). The process was repeated for the 30 samples in group $B$ and group $C$.

Since the materials and models were at a preset temperature in a water bath initially, any increase in temperature in the pulp chamber was attributed to the exothermic reaction of resin polymerization transmitted to the pulp chamber containing silver

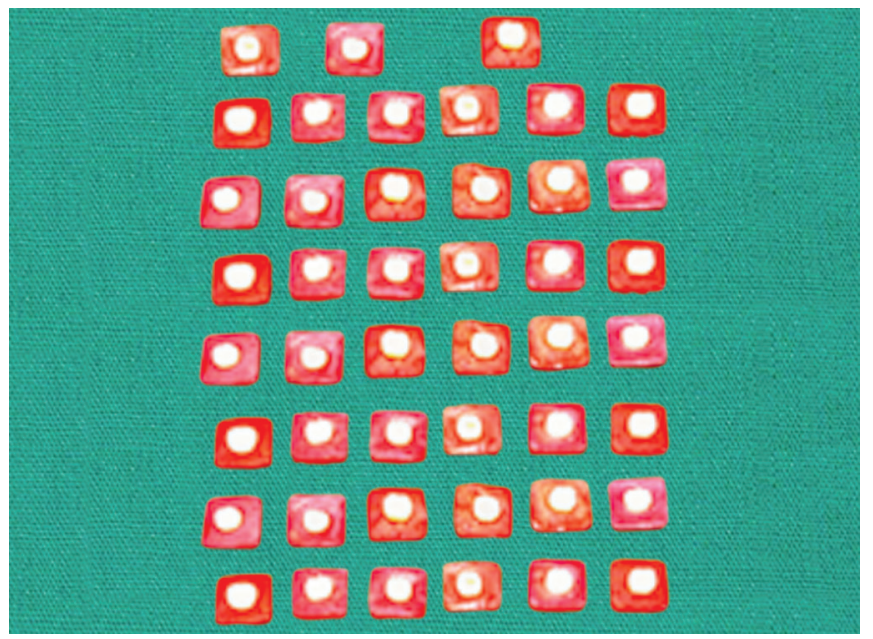

Fig. 2: Mandibular molars secured in autopolymerizing resin blocks 


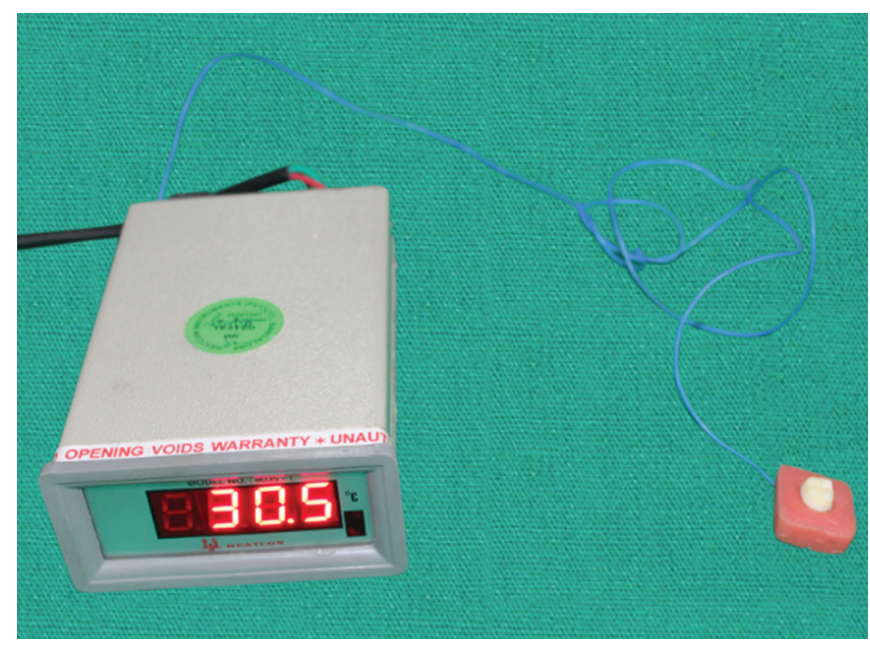

Fig. 3:Thermocouple unit with a digital indicator attached to the tooth

amalgam and the thermocouple. Intrapulpal temperature variations were recorded, tabulated, and statistically analyzed.

\section{Results}

All the description data are presented as mean and standard deviation. Analysis of variance (ANOVA) test was used for multiple group comparison.

Table 1 presents the intrapulpal temperature variations for three provisional materials. The temperature difference was significant between the three provisional materials used. The resultant temperature was highest among the three subgroups for all the three materials (group A-4.247 \pm 0.1552 , group B-2.273 \pm 0.1335 , and group $C-3.747 \pm 0.1356$ ).

The results of intragroup comparison (Table 2) showed statistically significant differences between the means of temperature rises of resins within each main group when considering means in pairs and analyzing these pairs one by one, i.e., on pairwise comparison, a statistically significant difference was detected while comparing means of temperature rises of resins with or without the insulating material. The mean difference in temperature was highest when subgroup I (control group) was compared with subgroup III (2 layers of DBA) for all the materials and lowest when subgroup I (control group) was compared with subgroup II (single layer of DBA).

The results of intergroup comparison (Table 3) also showed statistically significant differences between the means of

Table 1: Intrapulpal temperature variations for three provisional materials $(n=15)$

\begin{tabular}{llllll}
\hline Groups & Subgroups & Mean $^{*}$ & SD & fvalue & pvalue \\
\hline A & I & 4.247 & 0.1552 & 47.413 & $<0.001$ \\
& II & 3.993 & 0.1580 & & \\
& III & 3.680 & 0.1656 & & \\
B & I & 2.273 & 0.1335 & 56.301 & $<0.001$ \\
& II & 2.000 & 0.1512 & & \\
& III & 1.707 & 0.1534 & & \\
C & I & 3.747 & 0.1356 & 39.995 & $<0.001$ \\
& II & 3.520 & 0.1424 & & \\
& III & 3.227 & 0.1944 & & \\
\hline
\end{tabular}

"Temperature difference in ${ }^{\circ} \mathrm{C}$
Table 2: Results of intragroup comparison

\begin{tabular}{lllll}
\hline Groups & $\begin{array}{l}\text { Comparison between } \\
\text { subgroups }\end{array}$ & $\begin{array}{l}\text { Mean } \\
\text { difference (I-J) }\end{array}$ & Std error & pvalue \\
\hline A & I vs II & 0.253 & 0.0583 & $<0.001$ \\
& I vs III & 0.567 & 0.0583 & $<0.001$ \\
& II vs III & 0.313 & 0.0583 & $<0.001$ \\
B & I vs II & 0.273 & 0.0534 & $<0.001$ \\
& I vs III & 0.567 & 0.0534 & $<0.001$ \\
& II vs III & 0.293 & 0.0534 & $<0.001$ \\
C & I vs II & 0.227 & 0.0583 & $<0.001$ \\
& I vs III & 0.520 & 0.0583 & $<0.001$ \\
& II vs III & 0.293 & 0.0583 & $<0.001$ \\
\hline
\end{tabular}

temperature rises of the main groups of a particular subgroup when considering means in pairs and analyzing these pairs one by one, i.e., on pairwise comparison, a statistically significant difference was detected while comparing means of temperature rise with and without the insulating material in the presence of different provisional materials. The mean difference in temperature was highest when group A (PMMA) was compared against group B (bis-acrylic resin) and lowest when group $B$ (bis-acrylic resin) was compared with group C (DMMA).

On application of a single layer of DBA, no significant difference in intrapulpal temperature was found in comparison to the control group for all the three materials (group $A-0.253 \pm 0.0583$, group $B-0.273 \pm 0.0534$, and group C-0.227 \pm 0.0583$)$.

For the third test, 2 layers of DBA were applied and the resultant intrapulpal temperature was significantly lower in comparison to the control group (group $A-0.567 \pm 0.0583$, group $B-0.567 \pm$ 0.0534 , and group $C-0.520 \pm 0.0583$ ).

So the temperature rise varied according to the provisional restoration material used (PMMA $>$ DMMA $>$ bis-acrylic resin) $(p<0.001)$, and the single or double layer of DBA $(p<0.001)$. PMMA produced the highest intrapulpal temperature.

\section{Discussion}

Provisional restorations are fabricated to protect prepared teeth and adjacent gingiva until insertion of the final restoration. They also restore normal tooth function, prevent occlusal changes, and tooth migration. ${ }^{8}$ Provisional restorations should be the same as definitive restorations in all aspects, except for the material from which they are fabricated. ${ }^{9}$ Therefore, when considering the benefits of temporization, it cannot be avoided. Methods by which the process can be completed play a critical role.

Table 3: Results of intergroup comparison

\begin{tabular}{llccc}
\hline Subgroups & Comparison & $\begin{array}{l}\text { Mean } \\
\text { difference }(I-J)\end{array}$ & Std error & p value \\
\hline I & A vs B & 1.973 & 0.0518 & $<0.001$ \\
& A vs C & 0.500 & 0.0518 & $<0.001$ \\
II & B vs C & -1.473 & 0.0518 & $<0.001$ \\
& A vs B & 1.993 & 0.0550 & $<0.001$ \\
& A vs C & 0.473 & 0.0550 & $<0.001$ \\
III & B vs C & -1.520 & 0.0550 & $<0.001$ \\
& A vs B & 1.973 & 0.0628 & $<0.001$ \\
& A vs C & 0.453 & 0.0628 & $<0.001$ \\
& B vs C & -1.520 & 0.0628 & $<0.001$ \\
\hline
\end{tabular}


Various materials are available for fabrication of provisional restorations. These include various polymer-based PRMs, e.g., PMMA, ethyl methacrylate (EMA), vinyl methacrylate (VMA), bis-acrylic composite, visible-light polymerized composite, and butyl methacrylate. ${ }^{9}$ The chemical reaction of the polymerbased provisional material is an addition polymerization. As the polymerization proceeds, carbon-carbon double bonds ( $\pi$-bonds) are converted to new carbon-carbon single bonds ( $\sigma$-bonds). The carbon-carbon $\sigma$-bond has an energy of about $350 \mathrm{~kJ} / \mathrm{mol}$, and the carbon-carbon $\pi$-bond has $270 \mathrm{~kJ} / \mathrm{mol}$. The difference in energy between the two bonds, $80 \mathrm{~kJ} / \mathrm{mol}$, is emitted as exothermic heat. ${ }^{4}$

Castalnueva and $\operatorname{Tjan}^{10}$ reported that the temperature rise is lesser with the bis-GMA resin than the bis-acryl composite. It has been shown that the heat transferred to the pulp by PMMA resin and dimethacrylate-based materials ranged from 5.42 to $7.21^{\circ} \mathrm{C}^{7}$

This exothermic heat reaction may result in pulpal injury. According to Zach and Cohen, an intrapulpal temperature rise of $5.50^{\circ} \mathrm{C}(100 \mathrm{~F})$ in Rhesus Mecaca monkey resulted in $15 \%$ of pulpal vitality loss. ${ }^{11}$ Thermal damage results in various histopathological changes of the pulp, such as ectopic odontoblasts and their destruction, cellular degeneration, burn reactions at the periphery of the pulp including formation of blisters, coagulation of protoplasm, ${ }^{12}$ expansion of liquid in the dentinal tubules, and vascular injuries with generalized or localized tissue necrosis, resulting in acute inflammation of pulp, irreversible pulpitis, or pulp necrosis in severe cases. ${ }^{13-15}$

Dentinal walls exposed during tooth preparation are considered excellent nonconductors, because the thicker residual dentin has a greater insulating effect necessary to protect pulpal tissues from thermal injuries. ${ }^{10}$ Thus residual dentinal thickness plays a critical role in the protection of pulpal tissues from thermal injuries. ${ }^{8,16}$ However, tooth preparation may encroach on dentine based on the restorative material used and the amount of tooth structure present, prior to tooth preparation.

It has been suggested that desensitizing agents can be applied on prepared tooth surfaces to avoid complications during the interim stage, while the restoration is fabricated, and also before cementation. ${ }^{17}$ Cement base or dentin desensitizers were used in several studies as a physical barrier to achieve thermal insulation. ${ }^{18}$ The efficiency of cement base or desensitizing agent in providing thermal insulation is dependent on its thickness. ${ }^{19}$

The use of a thin layer of a resin-based dentin desensitizing agent may block dentinal tubules, which will subsequently reduce the thermal effects of external agents on dentin sensitivity and the pulp. Gurbulak et al. demonstrated that the sealing of dentinal tubules with polymeric resins reduced sensitivity and possibly the ingress of bacteria. ${ }^{8}$

In their study, Usumez et al. evaluated the effect of two dentin desensitizers on the pulp chamber temperature increase during the fabrication of provisional restorations by a direct method and found no significant difference in comparison to the control group. ${ }^{20}$ This is in contradiction to the results of our study, where application of a double layer of DBA resulted in a less intrapulpal temperature increase.

When a comparison was done between the single layer and double layer of DBA, a significant difference was found (group $A-0.313 \pm 0.0583$, group $B-0.293 \pm 0.0534$, and group $C-0.293 \pm$ 0.0583). The double layer of DBA was found to be more effective in terms of reduction in intrapulpal temperature. Thus a double layer of DBA can be used more effectively to prevent thermal injury to the tooth during the fabrication of provisional restorations. This is in agreement with the study done by Gurbulak et al. ${ }^{8}$

Three provisional materials, i.e., PMMA, bis-acrylic resin, and DMMA, were used in this study. The highest temperature was recorded for the PMMA provisional material $(4.247 \pm 0.1552)$, which is in agreement with previous studies. $2,7,13,14$

It should be noted that this is an in vitro study and therefore has some limitations. These include the use of amalgam in the pulp chamber, the use of acrylic resin to surround the prepared tooth, and in spite of uniformity attempted in the size of the molars, a discrepancy in dentinal thickness could have affected the heat transfer. The use of a water bath was deemed necessary to simulate intraoral conditions.

\section{Conclusion}

Within the limitations of this study, the following conclusions can be made:

- The intrapulpal temperature rise from greatest to least based on the provisional crown material used in the study was PMMA > DMMA > bis-acrylic resin.

- The intrapulpal temperature rise was greatest when provisional materials were used without any insulating material, i.e., control group.

- Application of a double layer of DBA results in a significantly lower intrapulpal temperature. Thus a double layer of DBA can be used during the fabrication of provisional restorations to protect the pulpal tissue from the polymerization heat of provisional materials.

\section{Clinical Significance}

Polymerization of materials used for the fabrication of provisional restorations is associated with an exothermic reaction. This temperature rise may result in thermal trauma to the dental pulp. Dentin bonding agent can be used to minimize the harmful effect.

\section{References}

1. American Prosthodontic Society. The Glossary of Prosthodontic Terms. J Prosthet Dent 2005;94(1):10-92. DOI: 10.1016/j.prosdent.2005.03.013.

2. Michalakis K, Pissiotis A, et al. Comparison of temperature increase in the pulp chamber during the polymerization of materials used for the direct fabrication of provisional restorations. J Prosthet Dent 2006;96:418-423. DOI: 10.1016/j.prosdent.2006.10.005.

3. Shillingburg HT, Hobo S, et al. Fundamentals of fixed prosthodontics, 3rd ed., Chicago quintessence; 1997. pp. 225-256.

4. Kim SH, Watts DC. Exotherm behaviors of the polymer-based provisional crown and fixed partial denture materials. Dent Mater 2004;20:383-387. DOI: 10.1016/j.dental.2003.11.001.

5. Grajower R, Shaharbani S, et al. Temperature rise in pulp chamber during fabrication of temporary self-curing resin crowns. J Prosthet Dent 1979;41:535-540. DOI: 10.1016/0022-3913(79)90088-X.

6. Moulding MB, Loney RW. The effect of cooling techniques on intrapulpal temperature during direct fabrication of provisional restorations. Int J Prosthodont 1991;4:332-336.

7. Moulding MB, Teplitsky PE. Intrapulpal temperature during direct fabrication of provisional restorations. Int J Prosthodont 1990;3 :299-304.

8. Gurbulak AG, Kilic K, et al. The effect of dentin desensitizer with different layers on thermal changes on the pulp during fabrication of provisional restoration. J Biomed Mater Res B 2009;91B:362-365. DOI: 10.1002/jbm.b.31410. 
9. Burns DR, Beck DA, et al. A review of selected dental literature on contemporary provisional fixed prosthodontic treatment: Report of the committee of research in fixed prosthodontics of the academy of fixed prosthodontics. J Prosthet Dent 2003;90:474-497. DOI: 10.1016/ S0022-3913(03)00259-2.

10. Castelnuovo J, Tjan AH. Temperature rise in pulpal chamber during fabrication of provisional resinous crowns. J Prosthet Dent 1997;78:441-416. DOI: 10.1016/S0022-3913(97)70057-X.

11. Zach L, Cohen G. Pulp response to externally applied heat. Oral Surg Oral Med Oral Path 1965;19:515-530. DOI: 10.1016/00304220(65)90015-0.

12. Langeland K, Langeland LK. Pulp reactions to crown preparation, impression, temporary crown fixation, and permanent cementation. J Prosthet Dent 1965;15:129-143. DOI: 10.1016/0022-3913(65)90073-9.

13. Nyborg $\mathrm{H}$, Brannstrom M. Pulp reaction to heat. J Prosthet Dent 1968;19:605-612. DOI: 10.1016/0022-3913(68)90262-X.

14. Driscoll CF, Woolsey G, et al. Comparison of exothermic release during polymerization of four materials used to fabricate interim restorations. J Prosthet Dent 1991;65:504-506. DOI: 10.1016/00223913(91)90289-9.
15. Goodis HE, White JM, et al. Measurement of temperature generated by visible light-cure lamps in an in-vitro model. Dent Mater 1989;5:230-234. DOI: 10.1016/0109-5641(89)90066-3.

16. Tjan $A H$, Grant $B E$, et al. Temperature rise in the pulp chamber during fabrication of provisional crowns. J Prosthet Dent 1989;62:622-626. DOI: 10.1016/0022-3913(89)90578-7.

17. Mausner IK, Goldstein GR, et al. Effect of two dentinal desensitizing agents on retention complete cast coping using four cements. J Prosthet Dent 1996;75:129-134. DOI: 10.1016/S0022-3913(96) 90088-8.

18. Akova T, Ozkomur A, et al. Intrapulpal heat generation during provisionalization: effect of desensitizer and matrix type. J Prosthodont 2008;17:108-113. DOI: 10.1111/j.1532-849X.2007.00257.x.

19. Voti ED, Phillips RW, et al. Thermal Diffusion through Amalgam and Various Liners. J Dent Res 1966;45(4):1184-1190. DOI: $10.1177 / 00220345660450042501$.

20. Usumez A, Ozturk AN, et al. The effect of dentin desensitizers on thermal changes in the pulp chamber during fabrication of provisional restorations. J Oral Rehabil 2004;31:579-584. DOI: 10.1111/j.1365-2842.2004.01281.x. 